\title{
Análisis y diseño de un molino eléctrico de granos económico para PYMES.
}

Analysis and design of a economic grain electric mill for smes.

Eugenia Mercedes Naranjo Vargas ${ }^{1}$., Javier Renato Moyano Arévalo ${ }^{2}$., Celin Abad Padilla Padilla ${ }^{3}$. \& Carlos José Santillán Mariño ${ }^{4}$.

Recibido: 17-02-2019 / Revisado: 19-02-2019 /Aceptado: 06-03-2019/ Publicado: 04-04-2019

\begin{abstract}
DOI: https://doi.org/10.33262/cienciadigital.v9i2.387

This document analyzes the design and construction of an electric mill in conjunction with a grain mixer, since in small farms there is a need to grind and mix grains, to optimally feed the birds or animals that require some grain type or balanced nutritional. The milling is built with a versatile and economical tool that facilitates the acquisition or manufacturing of the farmers dedicated to the production of animals.

In the development of the project different relationships are made between the variables involved in the grinding process of the grains such as, average size of each grain, maximum capacity of the machinery, mill time according to the type of grain and several factors that they directly or indirectly influence the execution of the project, explaining in a scientific and technical way the different phenomena that occur in the grinding process of the grains, besides guaranteeing the selection of materials that make the machine as economical as possible with a value Approximately \$137.40 US dollars, accessible to most SMEs.
\end{abstract}

Keywords: design, electric mill, grains, machinery, SMEs.

\section{Resumen}

En el presente documento hace un análisis al diseño y construcción de un molino eléctrico en conjunto con un mezclador de granos, ya que en pequeñas granjas existe la necesidad de moler y mezclar granos, para alimentar de manera óptima a

${ }^{1}$ Escuela Superior Politécnica de Chimborazo, Facultad de Mecánica, Carrera de Ingeniería Industrial. Riobamba, Ecuador, eugenia.naranjo@espoch.edu.ec

2 Escuela Superior Politécnica de Chimborazo, Facultad de Mecánica, Carrera de Ingeniería de Mantenimiento Industrial. Riobamba, Ecuador, javier.moyano@espoch.edu.ec

${ }^{3}$ Escuela Superior Politécnica de Chimborazo, Facultad de Mecánica, Carrera de Ingeniería Automotriz. Riobamba, Ecuador, celin.padilla@espoch.edu.ec

${ }^{4}$ Escuela Superior Politécnica de Chimborazo, Facultad de Mecánica, Carrera de Ingeniería Industrial. Riobamba, Ecuador, carlos.santillan@espoch.edu.ec 
las aves o animales que requieran algún tipo grano o balanceado alimenticio. El molido es construido con herramienta versátil y económica que facilite a los granjeros dedicados a la producción de animales su adquisición o fabricación.

En el desarrollo del proyecto se realiza diferentes relaciones entre las variables que intervienen en el proceso de molido de los granos tales como, tamaño promedio de cada grano, capacidad máxima de la maquinaria, tiempo de molino de acuerdo al tipo de grano y varios factores que influyen directa o indirectamente en la ejecución del proyecto, explicando de una forma científica - técnica los diferentes fenómenos que ocurren en el proceso de molido de los granos, además se garantiza la selección de materiales que hacen de la maquina lo más económica posible con un valor aproximado de $\$ 137,40$ dólares americanos, accesible para la mayoría de pymes.

Palabras clave: diseño, molino eléctrico, granos, maquinaria, pymes.

\section{Introducción.}

La actividad de moler y mezclar granos en la mayoría de granjas se lo realiza de forma manual, considerándose así una actividad que requiere de tiempo y de técnica para realizar el proceso. El principal problema es el tiempo y esfuerzo que lleva a la mano de obra el realizar el moler y mezclar granos, muchas veces sin obtener el resultado deseado, y es por ello que surge de la idea de la fabricación de un molino en conjunto de una mezcladora de granos que pueda ser utilizado por pequeños empresarios que desean mejorar la calidad de su producto, reducir los tiempos y esfuerzo que este tipo de actividad conlleva. (Solis, 2016)

Uno de los objetivos es llegar a los pequeños y medianos empresarios dedicados al área avícola o crianza de animales, para permitir que el tiempo y esfuerzo de producción se reduzca, manteniendo la calidad de los productos, mediante la correcta y responsable utilización de esta maquinaria que permitirá realizar el trabajo de molido de su materia prima. Este prototipo tiene ventajas tales como:

Seleccionar el tamaño de grano: como es común en los molinos tradicionales viene equipado con una perilla para poder seleccionar el grosor del grano, ya que en una granja avícola los pollos de semana de edad no tienen el mismo alimento que uno de 5 semanas por ende es importante seleccionar el tamaño de grano según la edad de los animales. (Díaz, 2012)

Reducción de tiempos y esfuerzos del operario: al realizar esta actividad de manera manual conlleva a una fatiga del operario por una mala postura, esto conlleva a que sea vulnerable a enfermedades como hernias, lumbalgias etc. Por tal motivo se suele tomar descansos periódicos lo cual hace que el proceso se vuelve lento y tedioso, implementado el motor eléctrico hará que el trabajo sea continuo y mucho más rápido que la manera tradicional. (Lefcovich, 2005) 
Aplicaciones: el molino no solo puede ser aplicado en la industria avícola o crianza de animales, también puede ser aplicado en la industria alimentaria ya que ciertos alimentos necesitan ser molidos y mezclados antes de llevar a cocción

Homogeneidad de mezcla: es vital importancia que los animales a recibir el alimento tengan correcta distribución de los alimentos, asegurando que cada nutriente llegue de manera equitativa a cada uno de los animales.

Fácil ensamblaje y transportación: como sabemos una máquina elaborada de piezas metálicas tiene la característica de ser pesada y de difícil transportación, por ende, se hará con elementos que permitan desmontar todo el prototipo en partes que permitan facilitar el transporte del mismo.

Esta máquina realizará 2 procesos que son moler y mezclar diferentes granos, para lo cual se analizará cada uno por separado para comprender mejor la utilidad de cada uno.

\section{Molido}

El molido de granos es una de las actividades previas permite agilizar (Carreira, s.f.), la reducción de tiempos, tratando siempre de mantener la calidad de la materia prima optimizando así también el espacio físico que ocupa una cantidad específica de granos la cual es otra de las variables a tomar en cuenta al momento de comercialización de un producto. (Hall, 1998). Uno de los diferentes tipos de molinos y sobre todo más utilizados es el molino para nixtamal la cual es una máquina agrícola, que se utiliza para llevar a cabo el proceso de molienda del maíz (SKS, 2014).

\section{Motor}

El motor es básicamente considerado como la parte sistemática de una máquina capaz de hacer funcionar un sistema, cuya función principal es trasformar algún tipo de energía ya sea eléctrica, eólica, solar u otras en energía mecánica para la realización de algún trabajo o actividad. $(\mathrm{R}, 1989)$

Para el funcionamiento del molino eléctrico se ha de utilizar una polea con un radio mínimo de acuerdo a la potencia del motor y a la velocidad del mismo, en este caso es de 2 HP y $1700 \mathrm{rpm}$ respectivamente, con dichos datos nos ubicamos en la siguiente tabla y determinamos el valor del radio que aproximadamente debe poseer la polea.

Tabla 1: Radios Mínimos en mm para la potencia del motor (INTERMEC)

\begin{tabular}{|c|c|c|c|c|}
\hline \multirow{2}{*}{$\begin{array}{c}\text { Caballos de fuerza } \\
(\text { HP) }\end{array}$} & \multicolumn{5}{|c|}{ RPM de Motor } \\
\cline { 2 - 5 } & $\mathbf{9 0 0}$ & $\mathbf{1 2 0 0}$ & $\mathbf{1 8 0 0}$ & $\mathbf{3 6 0 0}$ \\
\hline 0.50 & 65 & - & - & - \\
\hline 0.75 & 65 & 65 & - & - \\
\hline
\end{tabular}




\begin{tabular}{|c|c|c|c|c|}
\hline 1.00 & 65 & 65 & 60 & - \\
\hline 1.50 & 75 & 65 & 65 & 60 \\
\hline 2.00 & 75 & 65 & 65 & 65 \\
\hline 3.00 & 75 & 75 & 65 & 65 \\
\hline 5.00 & 100 & 75 & 75 & 65 \\
\hline 7.50 & 115 & 100 & 75 & 75 \\
\hline 10.00 & 115 & 115 & 100 & 75 \\
\hline 15.00 & 135 & 115 & 115 & 100 \\
\hline 20.00 & 155 & 135 & 115 & 115 \\
\hline 25.00 & 170 & 155 & 115 & 115 \\
\hline 30.00 & 170 & 170 & 135 & - \\
\hline 40.00 & 210 & 170 & 155 & - \\
\hline 50.00 & 230 & 210 & 170 & - \\
\hline 60.00 & 260 & 230 & 190 & - \\
\hline 75.00 & 260 & 260 & 230 & - \\
\hline 100.00 & 350 & 350 & 250 & - \\
\hline 125.00 & 380 & 350 & 280 & - \\
\hline 150.00 & 465 & 350 & - & - \\
\hline 200.00 & 550 & - & - & - \\
\hline 250.00 & - & - & - & - \\
\hline 300.00 & - & - & - & - \\
\hline
\end{tabular}

Fuente: INTERMEC S.A

\section{Mezclado}

En el proceso de mezclar diferentes granos ya sean molidos o no, es para dar una dieta balanceada a las aves u animales que lo requieran, un proceso de mezclado correctamente realizado hará que todos los animales consuman la misma cantidad de nutrientes. En este prototipo no solo se mesclará de manera idónea, sino que, evitará el esfuerzo y tiempo que esta actividad conlleva (Hous, 2014).

El objetivo principal es diseñar, construir e implementar un molino de granos y una mezcladora de los granos molidos unificando estas actividades en una sola maquinaria dirigida al sector avícola de la provincia de Chimborazo y de la ciudad de Riobamba mediante la utilización de materiales de calidad, en ámbitos de propiedades mecánicas, 
tiempos de vida útil y costos, además de entender y comprender el funcionamiento del mecanismo del molido y mezclado de la maquinaria desde un punto de vista mecánico y dinámico, además aplicar los diferentes conocimientos adquiridos a lo largo de la carrera universitaria en cuanto conocimiento científico-técnico se refiere, otro parámetro además de analizar los diferentes fenómenos físicos producidos por los diferentes elementos que conforman los elementos de la maquinaria, controlar el correcto funcionamiento de la maquinaria para un molido de forma constante y un mezclado homogéneo; identificar los diferentes costos directos e indirectos en la producción de un molino mezclador de granos

Identificar el impacto ambiental que posee laos elementos de la maquinaria, tales como motor, estructura y su funcionamiento, permitir la obtención de un producto final apropiado para la utilización de acuerdo a la necesidad del sector avícola.

\section{Metodología.}

El proceso tiene como metodología descriptiva aplicativa con una idea clara del proceso general de molido y mezclado el cual empieza al encender la maquinaria y al colocar el grano en la tolva para que este comience a un proceso de trituración, una vez terminado este proceso de trituración el grano pasa a la mezcladora, como continuación del proceso se debe colocar en la tolva el segundo tipo de grano a triturar, y así sucesivamente el número de granos que deseen ser molidos y pasen a la mezcladora cuando estén los diferentes tipos de granos previamente molidos en la mezcladora estos procederán a mezclarse obteniendo una mezcla homogénea, cuando termine el proceso de mezclado el producto caerá hacia un recipiente terminando así un ciclo. (Ríos, 2006)

Para la selección del tipo de correa que se utilizara en la máquina se debe tener en cuenta la potencia que soportará y la velocidad en rpm que la misma soportara. En este caso la potencia a soportar es la del motor de $2 \mathrm{HP}$ y una velocidad de $1700 \mathrm{rpm}$. Con dichos datos obtenidos del motor se procede a interpretar la siguiente tabla para una mejor selección del tipo de correa a elegir.

\section{Ecuación 1}

$$
\text { Transformación de HP a KW }=2 H P * \frac{745,7 \text { Watts }}{1 \mathrm{HP}} * \frac{1 \mathrm{Kw}}{1000 \mathrm{watts}}=1,49 \mathrm{KW}
$$

Se puede apreciar que con los datos obtenidos el tipo de correa a utilizar será correas del tipo A debido a que posee características como la de adaptarse firmemente al canal de la polea evitando posibles deslizamientos entre polea y correa, y es capaz de soportar velocidades altas (TheGatesRubberCompany)

Al seleccionar los rodamientos los cuales deben facilitar los movimientos radiales de un sistema, además de ser muy utilizados porque de igual forma ayudan a reducir la fricción existente entre la superficie de dos piezas ejes o elementos móviles. Otra de las funciones importantes son las de apoyar y guiar componentes que giran entre sí a más de transmitir fuerzas (NSK, 2018). 
Para el bastidor es importante seleccionar tubo cuadrado el cual posee beneficios de la utilización de tubo cuadrado es su facilidad al momento de realizar actividades (DISMETAL, 2018), como la de soldar y la de corte, moldeado y mecanizado moderados, ya que se trata de un material muy denso. (PLANES, 2018). Este tipo de tubos por lo general pueden estar fabricados de diferentes materiales, todo esto depende de la necesidad técnica y el presupuesto económico que se posea (ACEROBSV, 2018). El hierro, el acero, el cobre, el plomo y el PVC son algunos de los materiales más habituales (Merino, 2013). Para la unión de los elementos se realiza con electrodo 6011 posee revestimiento de tipo celulósico (INDURA, 2018).

El molino mezclador estará construido con la necesidad de facilitar el esfuerzo físico y factor tiempo que son perdidas de dinero en la industria (Céspedes, 2013), el manejo del molino mezclador será de manera sencilla gracias diseño elaborado donde el operario solo deberá pulsar el switch de encendido y este empezara su funcionamiento con una velocidad y potencia especificada para el tipo de grano que se vaya a moler, triturando el grano seguido del mezclador que nos permitirá tener una mezcla homogénea entre sí, esto permitiendo que la manipulación sea sencilla y sin riesgos en el operario, con esto reduciríamos cualquier peligro ambiental que la maquina pueda estar produciendo al ser utilizado en el ambiente siendo también esto uno de nuestros objetivos el cuidado y preservación del ambiente al no contaminarlo en ninguna área desde el momento de la construcción de nuestro proyecto hasta que este esté en funcionamiento (Chadán, 2011).

Su fácil manipulación sus ventajas económicas y el ahorro de tiempo son unos de los pocos verificadores positivos que tendría nuestro proyecto al ser tomado como una herramienta de uso satisfactorio en la industria de productos avícolas en la ciudad de Riobamba y el resto de la provincia de Chimborazo (Uribe, 2014).

\section{Resultados.}

Para el análisis de cálculo y selección de materiales se considera una carga de 1 quintal cada 10 minutos para una producción diaria, valorizadas con 8 horas de labor con una consideración total diaria 48 quintales, es decir tranquilamente se podría abastecer a 10 000 aves en una semana con esta cantidad. Dentro de la selección para la maquina es importante establecer que se requiere de un motor de $2 \mathrm{Hp}$, ya que la dureza de maíz y a su vez el tiempo de hora diaria que va a laborar es importante, por ende, para la selección del tipo de transmisión por consideración económica se establece el de correa, por su bajo costo, facilidad de instalación entre otras consideraciones.

Es importante considerar un bastidor y para ello el tipo de perfil ideal (DISMETAL, 2018), en el mercado nacional existen algunos proveedores tanto nacional como importando (Merino, 2013), a lo que se decidió seleccionar tubo cuadrado (PLANES, 2018).

Además de elaborar ángulos, de los que se considera una barra de metal plana, con un doblado de $90^{\circ}$, para formar elemento en L, que sirve para el marco estructural 
(ACEROBSV, 2018). Algunos son los elementos que se seleccionaron y que se detallará a continuación desde los datos del motor hasta la parte final.

\section{Datos del Motor}

Velocidad del Motor $($ VM $)=1700 \mathrm{rpm}$

Potencia del Motor $=2 \mathrm{HP}$

Radio del Motor $(\mathbf{r M})=1$ pulgada $=0,083 \mathrm{ft}$

Radio de la Polea $(\mathbf{r P})=9$ pulgadas $=0,747 \mathrm{ft}$

Radio del Eje $(\mathbf{r E})=0,5$ pulgadas $=0,415 \mathrm{ft}$

Cinemática Plana de un Cuerpo Rígido

Cálculo de velocidades y aceleraciones en el motor y poleas

Transformación de RPM a rad/s

$$
1700 \frac{\mathrm{rev}}{\mathrm{min}}=\frac{2 \pi \mathrm{rad}}{1 \mathrm{rev}} * \frac{1 \mathrm{~min}}{60 \mathrm{~s}}=178,02 \frac{\mathrm{rad}}{\mathrm{s}}
$$

Velocidad Angular de la Polea

Velocidad del Motor $(V M)=$ Velocidad de la Polea $(V P)$

$V M=V P$

$\omega M * r M=\omega P r P$

$\frac{\omega M * r M}{r P}=\omega P$

$\omega P=\frac{\omega M * r M}{r P}$

$\omega P=\frac{178,02 * 0,083}{0,747}$

$\omega P=19,79 \frac{\mathrm{rad}}{\mathrm{s}} \quad$ Ecuación 2

Velocidad de la Polea (rpm)

$V P=19,78 \frac{\mathrm{rad}}{\mathrm{s}} * \frac{1 \mathrm{rev}}{2 \pi \mathrm{rad}} * \frac{60 \mathrm{~s}}{1 \mathrm{~min}}=188,88 \frac{\mathrm{rev}}{\mathrm{min}}$

Relación de Transmisión Motor- Polea 
$i=\frac{\text { Velocidad del Motor }}{\text { Velocidad de la Polea }}$

Ecuación 3

$i=\frac{1700 \mathrm{rpm}}{188,88}$

$i=\frac{9}{1}$

La velocidad de la Polea es 9 veces menor que la del Motor

Velocidad del Eje con respecto a la Polea

$$
\begin{aligned}
& V E j e=w \text { Polea } * r \text { Eje } \quad \text { Ecuación } 4 \\
& V E j e=19,79 * 0,415 f t \\
& V E j e=8,21 \frac{f t}{s}
\end{aligned}
$$

Par de frenado del motor

Par de Frenado $=\frac{5252 * H P}{V} \quad$ Ecuación 5

Donde:

$\mathbf{H P}=$ Caballos de fuerza del Motor

$\mathbf{V}=$ Velocidad del Motor

Constante $=5252$

Datos:

$\mathbf{H P}=2 \mathrm{Hp}$

$\mathbf{V}=1700 \mathrm{rpm}$

Constante $=5252$

Par de Frenado $=\frac{5252 * 2}{1700}$

Par de Frenado $=\frac{10504}{1700}$

Par de Frenado $=6,17 \frac{\mathrm{lb}}{\mathrm{ft}}$

Potencia realizada por la fuerza par de frenado

Potencia $=F * V$

Ecuación 6

Donde:

$\mathbf{F}=$ Fuerza par de Frenado 
$\mathbf{V}=$ Velocidad del Motor

\section{Datos:}

$\mathbf{F}=1,45 \frac{l b}{f t}$

$\mathbf{V}=1700 \mathrm{rpm}$

Potencia $=F * V$

Potencia $=6,17 \frac{\mathrm{lb}}{f t} * 1700 \mathrm{rpm}$

Potencia $=10504 \frac{\mathrm{lb}}{\mathrm{ft}} \mathrm{rpm}$

Para transformar el resultado a HP se debe dividir el resultado obtenido para 5252.

Potencia $=\frac{10504}{5252}$

Potencia $=2 \mathrm{HP}$

\section{Aceleración en el motor y polea}

Debido a la velocidad constante que posee cada una de estas piezas la aceleración es cero o nula.

Con este cálculo se demostró que la potencia del motor es igual a la potencia realizada por la fuerza par del frenado del mismo (Hibbeler, 2014)

Una vez que se ha realizado los cálculos es necesario obtener un esquema del prototipo con todas sus partes.

Figura 1. Molino de grano

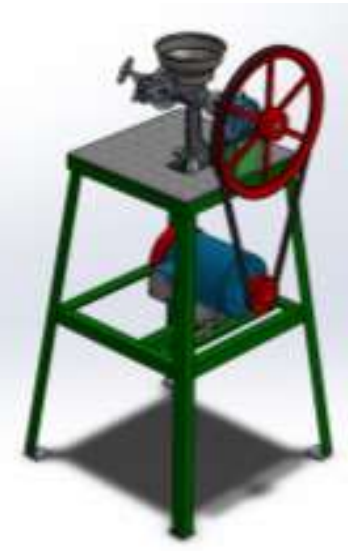

Fuente: Autores 
Con todos los datos obtenidos y considerando las partes que intervienen en la máquina se considera el presente informe de nuestro proyecto donde se desarrolló una tabla con cada una de los materiales que se utilizaron para su fabricación.

Tabla 2. Materiales utilizados

\begin{tabular}{|c|c|c|}
\hline Cantidad & Material & Características \\
\hline 7 & Tubo cuadrado & Tubo de pulgada $* 2 \mathrm{~mm}$ \\
\hline 4 & Angulos & La especificación de los ángulos es de $1 \frac{1}{2}$ pulgadas $* 2 \mathrm{~mm}$. \\
\hline 2 & Chumaceras & Chumaceras de piso de 1 pulgada. \\
\hline 1 & Motor $2 \mathrm{hp.}$ & La potencia del motor es de $1 \mathrm{hp.}$ \\
\hline 1 & Eje de trasmisión. & 40cm de largo $* 1$ pulgada de diametro. \\
\hline $1 / 2$ & Electrodos & Electrodos Aga 6011 \\
\hline 1 & Molino & Molino \\
\hline 2 & Tubo redondo & Tubo de diametro de 2 pulagadas. \\
\hline 1 & Banda para poleas. & Banda de $1 \frac{2}{2}$ tipo A \\
\hline 8 & Pernos & Pernos para ajuste mecánico. \\
\hline 8 & Arandelas. & Arandelas de protección para los pernos. \\
\hline
\end{tabular}

Fuente: Autores

Tabla 3. Materia prima

\begin{tabular}{|c|c|c|c|}
\hline Descripción & Cantidad & Unidad & Total \\
\hline Tubo cuadrado & 7 & $\mathrm{~m}$ & 9,1 \\
\hline Águlos & 4 & $\mathrm{Plg}$. & 6,8 \\
\hline Chumaceras de piso & 6 & $\mathrm{~mm}$ & 6 \\
\hline Motor 1 HP & 1 & $\mathrm{HP}$ & 25 \\
\hline Eje de Transmisión & 1 & $\mathrm{~m}$ & 15 \\
\hline Electrodos & $1 / 2$ & $\mathrm{~m}$ & 1,1 \\
\hline Molino & $1 / 2$ & $\mathrm{plg}$ & 10 \\
\hline Tubo redondo & 2 & $\mathrm{~m}$ & 5,4 \\
\hline Correa para poleas & 1 & $\mathrm{~m}$ & 3 \\
\hline Pernos & 8 & $\mathrm{u}$ & 2,8 \\
\hline Arandelas & 8 & $\mathrm{~mm}$ & 1,6 \\
\hline
\end{tabular}

Fuente: Autores.

Tabla 4. Mano de obra directa

\begin{tabular}{|c|c|c|c|}
\hline Descripción & Cantidad & Duración & Total \\
\hline Armado de la etructura metálica. & 1 & 5 horas & 10 \\
\hline Montaje del molino. & 1 & 3 horas & 5 \\
\hline Montaje del motor. & 1 & 4 horas & 5 \\
\hline Armado de los accesorios de seguridad. & 1 & 2 horas & 5 \\
\hline
\end{tabular}

Fuente: Autores. 
Tabla 4. Costos indirectos

\begin{tabular}{|c|c|c|c|c|}
\hline Costo indirecto & Descripción & Cantidad & Duración & Total \\
\hline Soldeo & Energía uilizada para soldar. & 1 & 2 horas & 1 \\
\hline Transporte & $\begin{array}{c}\text { Movilización para el traslado de } \\
\text { la materia prima entre otros. }\end{array}$ & 7 & 1 hora & 5,6 \\
\hline
\end{tabular}

Fuente: Autores.

\section{Total, de Costo de Producción}

A continuación, se hace una breve valoración de cada uno de los costos realizados en la presente investigación detallado a continuación.

Total, costo de producción $=$ costo de materia prima + costo de mano de obra directa + costos indirectos + valor agregado (personal)

Total, costo de producción $=(85,8+25,00+6,60+20)$ dólares

Total, costo de producción= 137, 40 dólares

\section{Conclusiones.}

- Se obtiene una reducción de velocidad del motor mediante el uso de una polea de mayor radio pues mediante cálculos de logro determinar que la velocidad de la polea es de 188, 88 rpm obteniéndose además una relación de transmisión motor polea de 9:1 lo cual facilita el proceso de molido de granos.

- Para un par de motor de 6, $17 \mathrm{lb} / \mathrm{ft}$, se establece una potencia de $2 \mathrm{HP}$.

- Se garantiza la reducción del tamaño de grano dependiendo de las necesidades de los operarios.

- El grano que es el resultado final del proceso posee mejor calidad permitiendo un mejor rendimiento desde el punto zootécnico en animales pues su digestividad es mucho más fácil.

- Mediante los cálculos realizados el uso de poleas reduce o aumentan considerablemente las velocidades que poseen cada uno de los elementos que conformen el sistema de poleas como lo es motor, ruedas y ejes, únicamente se debe conocer la aplicación que se desea darle a la potencia y velocidad que ejerce el motor y en que mecanismo se desea implementa. 


\section{Referencias Bibliográficas}

ACEROBSV. (2018). ACEROBSV. Obtenido de ACEROBSV: https://acerobsv.com/angulos-estandar.html

Aldana, S., Vereda, F., Hidalgo-Alvarez, R., \& de Vicente, J. (2016). Facile synthesis of magnetic agarose microfibers by directed selfassembly. Polymer, 93, 61-64.

aprendemostecnologia.org. (2015). Obtenido de Mecanismos de transmisión del movimiento: https://aprendemostecnologia.org/maquinas-ymecanismos/mecanismos-de-transmision-del-movimiento/

Bhat, S., Tripathi, A., \& Kumar, A. (2010). Supermacroprous chitosan-agarose-gelatin cryogels. in vitro characterization and in vivo assesment for cartilage tissue engineering. Journal of the Royal Society Interface, 1-15.

Bossis, G., Marins, J., Kuzhir, P., Volkova, O., \& Zubarev, A. (2015). Functionalized microfibers for field-responsive materials and biological applications. Journal of Intelligent Material Systems and Structures, 1-9.

Carreira, I. (s.f.). Simbiosis S Coop. Galega. Obtenido de https://www.cooperativasimbiosis.com/harinas/

Céspedes, P. (2013). Diseño y construcción de un molino artesanal para molienda de granos secos de capacidad $250 \mathrm{Kg} / \mathrm{h}$.

Chadán, J. C. (2011). ESTUDIO DE UN SISTEMA DOSIFICADOR DE GRANOS EN LA TOLVA DE UN MOLINO DE PIEDRA PARA OPTIMIZAR EL PROCESO DE MOLIENDA DE CEBADA Y MAÍZ.

Comunicación, C. ( 25 de enero de 2017). El Productor. Obtenido de http://elproductor.com/articulos-tecnicos/articulos-tecnicos-saludanimal/alimentacion-del-ganado-y-sistemas-de-pastoreo/

contributors, E. (8 de febrero de 2012). Picadora de forrajes. Obtenido de https://www.ecured.cu/Picadora_de_forrajes

Cortés, J., Puig, J., Morales , J., \& Mendizábal, E. (2011). Hidrogeles nanoestructurados termosensibles sintetizados mediante polimerización en microemulsión inversa. Revista Mexicana de Ingeniería Química., 10(3), 513-520.

Dias, A., Hussain, A., Marcos, A., \& Roque, A. (2011). A biotechnological perspective on the application of iron oxide magnetic colloids modified with polysaccharides. Biotechnology Advances 29 , 29, 142-155.

Díaz, P. (2012). El molino perfecto . Obtenido de 
http://www.ucsj.edu.mx/claustronomia/index.php/investigacion/223-el-molidoperfecto-del-cafe

DISMETAL. (2018). DISMETAL. Obtenido de DISMETAL: https://dismetal.ec/productos/planchas/galvanizadas

Estrada Guerrero, R., Lemus Torres, D., Mendoza Anaya, D., \& Rodriguez Lugo, V. (2010). Hidrogeles poliméricos potencialmente aplicables en Agricultura. Revista Iberoamericana de Polímeros, 12(2), 76-87.

García-Cerda, L., Rodríguez-Fernández, O., Betancourt-Galindo, R., Saldívar-Guerrero, R., \& Torres-Torres, M. (2003). Síntesis y propiedades de ferrofluidos de magnetita. Superficies y Vacío., 16(1), 28-31.

Hall, C. W. (1998). Equipo para procesamiento de Productos Avícolas . Lima.

Hibbeler, R. (2014). Mecánica Vectorial para Ingenieros, Dinámica .

Hous, B. (2014). El sitio Avicola. Obtenido de http://www.elsitioavicola.com/articles/2967/granulometraa-importancia-deltamaao-de-las-particulas-de-alimento-en-las-ponedoras-2/

Ilg, P. (2013). Stimuli-responsive hydrogels cross-linked by magnetic nanoparticles. Soft Matter, 9, 3465-3468.

INDURA. (2018). INDURA. Obtenido de INDURA: http://www.cryogas.com.co/Descargar/INDURA\%206011\%20\%E2\%80\%93\%2 0AWS $\% 20 \mathrm{E}-$

6011?path $=\% 2$ Fcontent $\% 2$ Fstorage $\% 2 F c o \% 2 F b i b l i o t e c a \% 2 F d b 54 d 3924 c 364 f 2$ c8e2c3377b65f8ba9.pdf

Lara, C. (2012). DISEÑO DE UN MOLINO DE RODILLOS PARA MOLIENDA DE GRANOS DE ALIMENTOS DESTINADOS AL SECTOR DEL CESTTAESPOCH. Riobamba. Obtenido de http://dspace.espoch.edu.ec/bitstream/123456789/1982/1/96T00161.pdf

Lefcovich, M. (2005). Herramientas y reducción de tiempos de producción. Obtenido de https://www.gestiopolis.com/smed-herramientas-reduccion-tiempos-produccion/ Lewitus, D., Branch, J., Smith, K., Callegari, G., Kohn, J., \& Neimark, A. (2011). Biohybrid carbon nanotube/agarose fibers for neural tissue engineering. Advanced Functional Materials, 21, 2624-2632.

Lin, Y.-S., Huang, K.-S., Yang, C.-H., Wang, C.-Y., Yang, Y.-S., Hsu, H.-C., . . Tsai, C.-W. (2012). Microfluidic synthesis of microfibers for magnetic-responsive controlled drug release and cell culture. PLoS ONE, 7(3), 1-8. 
Merino, J. P. (2013). DEFINICION DE . Obtenido de DEFINICION DE : https://definicion.de/tubo/

Nelly, C. N. (2013). ejores prácticas para mejorar la eficiencia de la producción y el trabajo. Obtenido de https://www.gestiopolis.com/mejores-practicas-paramejorar-la-eficiencia-de-la-produccion-y-el-trabajo/

NSK. (2018). NSK. Obtenido de NSK: https://www.nskeurope.es/que-es-un-rodamiento152.htm

PLANES, F. (03 de 10 de 2018). PLANES. Obtenido de PLANES: https://ferrosplanes.com/tubo-cuadrado-caracteristicas-fabricacion-corte-uso/

R, S. n. (10 de 12 de 1989). Maquinas electricas. Obtenido de WIKIPEDIA: https://es.wikipedia.org/wiki/Motor

Ríos, A. (2006). Tractores e implementos. Instituto de Investigaciones de Ingeniería Agrícola. Obtenido de https://www.ecured.cu/Molino_de_granos

Ruiz Estrada, G. (2004). Desarrollo de un Sistema de liberación de fármacos basado en nanopartículas magnéticas recubiertas con Polietilénglicol para el tratamiento de diferentes enfermedades. Madrid: Universidad Autónoma de Madrid. Departamento de Física Aplicada.

SKS. (14 de JUNIO de 2014). WIKIPEDIA. Obtenido de WIKIPEDIA: https://es.wikipedia.org/wiki/Molino_de_nixtamal

Smolenky, I. (11 de 12 de 1984). Maquinas electricas . moscú: Mir. Obtenido de WIKIPEDIA: https://es.wikipedia.org/wiki/Polea

Solis, F. (2016). Sitio Argentino de producción Animal . Obtenido de El sitio Avicola.com: http://www.produccionanimal.com.ar/produccion_aves/produccion_avicola/154Importancia_tamanio_particulas.pdf

Song, J., King, S., Yoon, S., Cho, D., \& Jeong, Y. (2014). Enhanced spinnability of narbon nanotube fibers by surfactant addition. Fiberes and Polymers, 15(4), 762766.

Tartaj, P., Morales, M., González-Carreño, T., Veintemillas-Verdaguer, S., \& Serna, C. (2005). Advances in magnetic nanoparticles for biotechnology applications. Journal of Magnetism and Magnetic Materials, 290, 28-34.

TheGatesRubberCompany. (s.f.). Heavy Duty V-Belt Drive Design Manual. Denver, Colorado.

Uribe, J. (2014). Desarrollo Consturción y pruebas de funcionamiento de un prototipo de 
moino de martillos. Floridablanca.

Wulff-Pérez , M., Martín-Rodriguez, A., Gálvez-Ruiz, M., \& de Vicente, J. ( 2013 ). The effect of polymer surfactant on the rheological properties of nanoemulsions. Colloid and Polymer Science, 291, 709-716.

Zamora Mora, V., Soares, P., Echeverria, C., Hernández , R., \& Mijangos, C. (2015). Composite chitosan/Agarose ferrogels for potential applications in magnetic hyperethermia. Gels., 1, 69-80.

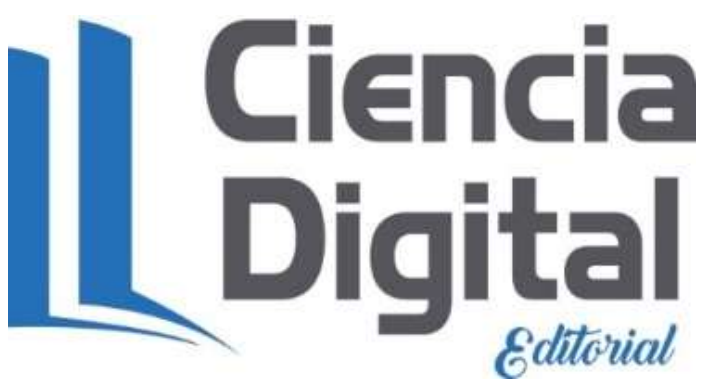




\section{Para citar el artículo indexado.}

Naranjo E., Moyano J., Padilla C. \& Santillán C. (2019). Análisis y diseño de un molino eléctrico de granos económico para PYMES. Revista electrónica Ciencia Digital 3(2), 236-251.

\section{Liencia}

El artículo que se publica es de exclusiva responsabilidad de los autores y no necesariamente reflejan el pensamiento de la Revista Ciencia Digital.

El artículo queda en propiedad de la revista y, por tanto, su publicación parcial y/o total en otro medio tiene que ser autorizado por el director de la Revista Ciencia Digital.
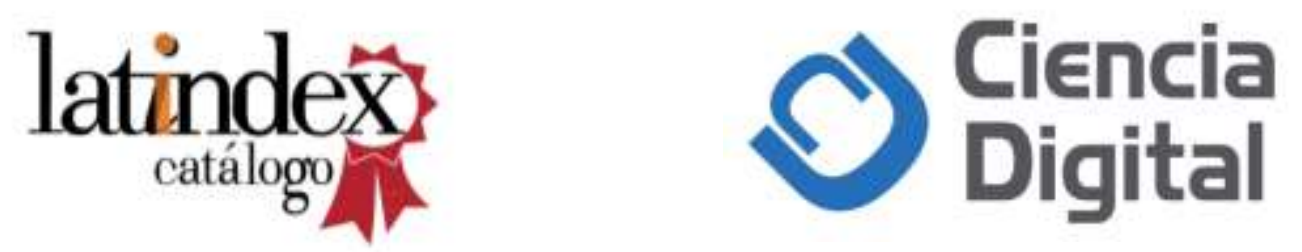